Karen CRISTine AbRÃo

Rossana Pulcineul Viera Francisco ${ }^{2}$

MARCELO ZUGAB ${ }^{3}$

Editorial

\section{Alterações cardiotocográficas após analgesia obstétrica combinada raqui-peridural}

\author{
Changes in cardiotochography following combined spinal-epidural \\ labor analgesia
}

Correspondência:

Karen Cristine Abrão Disciplina de Obstetrícia/Divisão de Clinica Obstétrica Hospital das Clinicas da Faculdade de Medicino da Universidade de São Paulo

Avenida Doutor Enéas de Carvalho Aguiar, $255,10^{\circ}$ andar sala 10.086 - Cerqueira César CEP 05403-000 - São Paulo/SP

E-mail: karenabrao@yahoo.com Fone/Fax: (11) 3069-6209
As alterações cardiotocográficas observadas durante o trabalho de parto conduzido sob analgesia obstétrica constituem um desafio para obstetras e anestesiologistas. Apesar do grande avanço dessas técnicas para minimizar sua interferência com a evolução do trabalho de parto e manter o bem-estar fetal, ainda existem dúvidas sobre o significado e o manejo das alterações da frequência cardíaca fetal (FCF) subsequentes a esses procedimentos ${ }^{1,2}$. Em particular, a técnica combinada raquiperidural, também conhecida no Brasil como duplo bloqueio, tem sido associada em diversos estudos a maior frequência de anormalidades cardiotocográficas, especialmente desacelerações prolongadas e bradicardia fetal, comparativamente à tradicional analgesia peridural ${ }^{3,4}$. A técnica combinada recebeu destaque nos últimos anos devido à qualidade e rapidez do alívio proporcionado à dor, além da redução no bloqueio motor, permitindo a deambulação durante o trabalho de parto ${ }^{5}$. Alguns centros no Brasil e no mundo adotaram a técnica como escolha quando a analgesia é iniciada na segunda fase do trabalho de parto e necessita ser rápida, uma vez que em menos de cinco minutos atinge-se grande alívio da dor, mesmo em mulheres com cervicodilatação completa ${ }^{6}$.

Uma das hipóteses aventada para explicar a associação entre o bloqueio combinado e as alterações cardiotocográficas sugere que o rápido alívio da dor obtido com a analgesia combinada levaria a um desbalanço transitório nos níveis maternos de catecolaminas, resultando em aumento do tônus uterino - hipertonia que poderia culminar em desacelerações ou bradicardia fetal ${ }^{7}$.

Recente publicação avaliou o efeito da analgesia obstétrica sobre o tônus uterino em 77 parturientes submetidas aleatoriamente à analgesia combinada raquiperidural ou à analgesia peridural tradicional, ambas com adição de opioide (sufentanil), confrontando os resultados do tônus uterino com os da cardiotocografia. As aferições do tônus uterino basal foram realizadas por meio de tocodinamometria interna, com cateteres de pressão intraamnióticos, durante os primeiros 15 minutos da analgesia. Foram incluídas apenas pacientes que receberam analgesia até $7 \mathrm{~cm}$ de cervicodilatação ${ }^{8}$. Observou-se aumento simultâneo do tônus uterino em 11 dos 13 casos que apresentaram alterações cardiotocográficas no grupo de analgesia combinada $(n=41)$ e apenas um de dois casos no grupo peridural $(n=36)$. Todas as pacientes que apresentaram alterações da cardiotocografia em ambos os grupos tiveram

Disciplina de Obstetrícia do Departamento de Obstetrícia e Ginecologia da Faculdade de Medicina da Universidade de São Paulo - USP - São Paulo (SP), Brasil.

' Professora do Departamento de Obstetrícia e Ginecologia da Faculdade de Medicina da Universidade de São Paulo - USP - São Paulo (SP), Brasil.

2 Professora Doutora do Departamento de Obstetrícia e Ginecologia da Faculdade de Medicina da Universidade de São Paulo - USP - São Paulo (SP), Brasil.

3 Professor Titular de Obstetrícia do Departamento de Obstetrícia e Ginecologia da Faculdade de Medicina da Universidade de São Paulo - USP - São Paulo (SP), Brasil. 
resolução espontaneamente ou com medidas tocolíticas gerais ${ }^{8}$. O estudo citado encontrou ainda associação entre a velocidade de alívio da dor pela analgesia (aferida por escala visual analógica de dor) e a probabilidade de ocorrência de anormalidades cardiotocográficas, observando que quanto mais rápido for o alívio da dor, maior será a probabilidade de ocorrência de alterações da $\mathrm{CTG}^{8}$. Tais achados confirmam resultados de estudos anteriores, que propunham o rápido alívio da dor como possível fator envolvido na ocorrência de bradicardia após o duplo bloqueio, sendo o primeiro estudo encoberto e randomizado a confirmar a fisiopatologia previamente proposta por diversos autores ${ }^{8}$.

O ensaio calculou um "number needed to harm" de 4,2 (IC95\%=2,6-10,2), o que significa que o risco de desacelerações prolongadas ou bradicardia fetal associada a aumento de tônus uterino após a analgesia combinada foi de aproximadamente uma a cada quatro mulheres. Considerando-se esses valores, pode-se recomendar conduta cautelosa durante os primeiros minutos de uma analgesia combinada. Todas as parturientes devem ser cuidadosamente monitorizadas, em especial durante a primeira meia hora após a analgesia. A administração de ocitocina nesse período crítico merece atenção, pois pode potencializar o efeito uterotônico da analgesia ${ }^{9}$. Além disso, deve-se ponderar a indicação dessa técnica em pacientes com hiperatividade uterina prévia ou mesmo em fetos com sabido comprometimento da reserva de oxigênio ${ }^{8}$.

Apesar das anormalidades cardiotocográficas observadas parecerem muitas vezes alarmantes aos olhos dos profissionais que as acompanham, a literatura até o presente tem sido unânime em relação ao bom prognóstico neonatal dos casos com tais alterações após a analgesia. Nos vários estudos, no entanto, não se observou piora nos índices de Apgar ou do pH de cordão umbilical entre os casos com bradicardia fetal em comparação com aqueles que não apresentaram essa alteração após a analgesia ${ }^{2,7,10}$. No entanto, mesmo com resultados neonatais normais, há de se considerar que as desacelerações prolongadas da FCF são eventos geradores de ansiedade na sala de parto para obstetras, anestesistas e pacientes e que o manejo inadequado dos casos pode levar a intervenções obstétricas de urgência desnecessárias ${ }^{1}$.

Estudos dessa natureza envolvem vícios potenciais, uma vez que parturientes que solicitam analgesia regional em detrimento de outras técnicas, em geral, são as que têm os partos mais dolorosos, morosos e possivelmente mais associados à distocias, fatores reconhecidamente associados à hiperatividade uterina e à alterações da $\mathrm{FCF}^{11-13}$. Todavia, esses potenciais vieses de qualquer estudo relacionado à analgesia obstétrica têm sua relevância reduzida quando se pondera que aquelas pacientes que requerem analgesia regional durante o parto constituem o verdadeiro grupo de interesse desse tipo de pesquisa, pois na prática clínica, o que se deseja é compreender a fisiologia do parto e suas complicações nos casos que necessitam de analgesia, para futuramente se desenvolverem estratégias de manejo ideal dessas mulheres e não simplesmente evitar que elas recebam analgesia ${ }^{11}$.

Vive-se, nos dias de hoje, interessante paradigma em relação à analgesia obstétrica ${ }^{14}$. Ela já foi considerada um dos fatores que contribuíram para o aumento das taxas de cesáreas ao redor do mundo todo, pela interferência na evolução natural do trabalho de parto. Recentes estudos têm mudado esse panorama, com novas evidências de que as doses e técnicas empregadas atualmente não prejudicam a progressão do parto como se pensava anteriormente ${ }^{15,16}$. Considerando-se que uma das causas da cesárea a pedido, tão frequente no Brasil, é o medo da dor do parto, a analgesia obstétrica, inversamente ao observado nas décadas anteriores, pode passar a contribuir para o aumento das taxas de partos vaginais, por livrar a mulher moderna do fantasma da dor.

Finalmente, é necessário enfatizar que os recentes avanços no campo da analgesia obstétrica - entre eles, o bloqueio combinado - foram insuficientemente estudados ${ }^{17}$. Embora a literatura médica seja concordante em afirmar que os resultados perinatais de fetos que apresentaram alterações cardiotocográficas após a analgesia não são diferentes dos resultados de fetos que não os apresentaram, mais investigações no campo da fisiologia da anestesia obstétrica são necessários para melhor compreensão dos seus efeitos no progresso do trabalho de parto e no bem-estar fetal. Tais questões são de fundamental importância para que a condução do trabalho de parto, com todos os recursos que a obstetrícia moderna tem a oferecer, seja embasada em evidências científicas.

\section{Referências}

1. Clarke VT, Smiley RM, Finster M. Uterine hyperactivity after intrathecal injection of fentanyl for analgesia during labor: a cause of fetal bradycardia? Anesthesiology. 1994;81 (4):1083.

2. Van de Velde $M$, Vercauteren $M$, Vandermeersch $E$. Fetal heart rate abnormalities after regional analgesia for labor pain: the effect of intrathecal opioids. Reg Anesth Pain Med. $2001 ; 26(3): 257-62$.

3. Van de Velde M. Neuraxial analgesia and fetal bradycardia. Curr Opin Anaesthesiol. 2005;18(3):253-6. 
4. Van de Velde M, Teunkens A, Hanssens M, Vandermeersch E, Verhaeghe J. Intrathecal sufentanil and fetal heart rate abnormalities: a doubleblind, double placebo-controlled trial comparing two forms of combined spinal epidural analgesia with epidural analgesia in labor. Anesth Analg. 2004;98(4): 1 153-9.

5. Nelson KE, Rauch T, Terebuh V, D'Angelo R. A comparison of intrathecal fentanyl and sufentanil for labor analgesia. Anesthesiology. 2002;96(5): 1070-3.

6. Albright GA, Forster RM. The safety and efficacy of combined spinal and epidural analgesia/anesthesia $(6,002$ blocks) in a community hospital. Reg Anesth Pain Med. 1999;24(2):117-25.

7. Cohen SE, Cherry CM, Holbrook RH Jr, el-Sayed YY, Gibson RN, Jaffe RA. Intrathecal sufentanil for labor analgesia: sensory changes, sideeffects and fetal heart rate changes. Anesth Analg. 1993;77(6):1 155-60.

8. Abrão KC, Francisco RP, Miyadahira S, Cicarelli DD, Zugaib M. Elevation of uterine basal tone and fetal heart rate abnormalities after labor analgesia: a randomized controlled trial. Obstet Gynecol. 2009;1 13(1):41-7.

9. Holdcroft A, Dob D. Regional analgesia for labour and fetal distress: culprit or innocent bystander? Int J Obstet Anesth. 2003; 12(3):153-5.

10. Vercauteren M, Bettens K, Van Springel G, Schols G, Van Zundert J. Intrathecal labor analgesia: can we use the same mixture as is used epidurally? Int J Obstet Anesth. 1997;6(4):242-6.

11. Gaiser RR. Labor epidurals and outcome. Best Pract Res Clin Anaesthesiol. 2005;19(1):1-16.

12. Miller AC. The effects of epidural analgesia on uterine activity and labor. Int J Obstet Anesth. 1997;6(1):2-18.

13. Riley ET, Vogel TM, El-Sayed YY, Meyer PB, Cohen SE. Fetal heart rate after combined spinal epidural - Selection bias may contrivute to incidence of fetal bradycardia. Anesthesiology. 1999;90 Suppl 4A:A40.

14. Hughes SC. Obstetrical anesthesia: the challenge of living in interesting times. ASA Newsl. 2006;70(8):1-5.

15. Ohel G, Gonen R, Vaida S, Barak S, Gaitini L. Early versus late initiation of epidural analgesia in labor: does it increase the risk of cesarean section? A randomized trial. Am J Obstet Gynecol. 2006;194(3):600-5.

16. Wong CA, Scavone BM, Peaceman AM, McCarthy RJ, Sullivan JT, Diaz NT, et al. The risk of cesarean delivery with neuraxial analgesia given early versus late in labor. N Engl J Med. 2005;352(7):655-65.

17. Eisenach JC. Obstetric anesthesia: what have you done for us lately? Anesthesiology. 1999;91 (4):907-8. 